\title{
A VIDEO CAMERA ROAD SIGN SYSTEM OF THE EARLY WARNING FROM COLLISION WITH THE WILD ANIMALS
}

Slavomir MATUSKA ${ }^{1^{*}}$, Robert HUDEC ${ }^{2}$, Patrik KAMENCAY ${ }^{3}$, Tibor TRNOVSZKY ${ }^{4}$

${ }^{1}$ Department of Telecommunications and Multimedia, Faculty of Electrical Engineering, University of Žilina, Univerzitná 8215/1, 01026 Žilina, Slovakia.

2 Department of Telecommunications and Multimedia, Faculty of Electrical Engineering, University of Žilina, Univerzitná 8215/1, 01026 Žilina, Slovakia.

${ }^{3}$ Department of Telecommunications and Multimedia, Faculty of Electrical Engineering, University of Žilina, Univerzitná 8215/1, 01026 Žilina, Slovakia.

4 Department of Telecommunications and Multimedia, Faculty of Electrical Engineering, University of Žilina, Univerzitná 8215/1, 01026 Žilina, Slovakia.

corresponding author: slavomir.matuska@fel.uniza.sk.

\begin{abstract}
This paper proposes a camera road sign system of the early warning, which can help to avoid from vehicle collision with the wild animals. The system consists of camera modules placed down the particularly chosen route and the intelligent road signs. The camera module consists of the camera device and the computing unit. The video stream is captured from video camera using computing unit. Then the algorithms of object detection are deployed. Afterwards, the machine learning algorithms will be used to classify the moving objects. If the moving object is classified as animal and this animal can be dangerous for safety of the vehicle, warning will be displayed on the intelligent road sings.
\end{abstract}

\section{Keywords:}

Animal detection and recognition; Video camera; Background subtraction; Road sign system; Vehicle collision.

\section{Introduction}

For the long period of time, there were many efforts in the traffic monitoring and the possible hazardous situation detection and early warning in the road traffic [1]. With the development of the high speed computers and CCTV cameras, this area are became even more popular between researcher and nowadays, there have been developed a lot of the systems which help prevent vehicle from crash in the hazardous situations. These systems together can create intelligent transportation system in the cities or in the particular areas. Their task is to enhance road safety by assisting drivers in their tasks. In [2], the improved traffic sign detection and recognition system was introduced. They developed two new modules: vision-module for sign detection and recognition and transmission module (TM) for communication between the cars. The main idea of the TM was sharing information with others cars in the area. The problem of the animal-vehicle collision (AVC) is a challenging problem and many papers were focusing on the preventing of the AVC. An efficient animal detection system for smart cars using cascaded classifiers was introduces in [3]. They equipped the cars with smart system with camera, which is able to detect animals around the roadways and warn the drivers. Their system works in two stages. First, LBP-adaboost algorithm is used to detect animals and then, HOG-SVM classifier is used to identify only the moose objects. According to their results, the success rate of the moose classification was more than $83 \%$. The state-of-art vehicle mounted night vision animal detection system was developed and presented in [4]. Their system is currently used by Audi, BMW and Daimler and is based on the cascade boosting concept which is robust to occlusion, pose and scale variations. Their system can detect animals up to 200 meters away from the car while generating very few false warning. A large animal detection and continuous traffic monitoring on highways was published in [5]. They used a $360^{\circ}$ - scanning radar to monitor a stretch of highway. The innovative analysis and classification techniques, which were used, enable the system to track large animals even in the harsh weather condition. 


\section{Proposed system solution}

In our system solution, we were considering the fact, that the most of the cars don't have intelligent camera system or mounted night vision animal detection system, which was presented in $[3,4]$. Therefore, we were focusing on developing a universal camera road sign system of early warning from collision with the wild animals, which can help every driver on the road. The system consists of camera modules placed down the particularly chosen route and the intelligent road signs. The camera module consists of camera device and computing unit. The video stream is captured from video camera using computing unit. Then the algorithms of object detection are deployed. Afterwards, the machine learning algorithms are used to classify the moving objects. If the moving objects inside the monitored area are classified as animal and this animal can be dangerous for safety of the vehicle, warning will be displayed on the intelligent road sings. The proposed system solution is shown in Fig. 1.

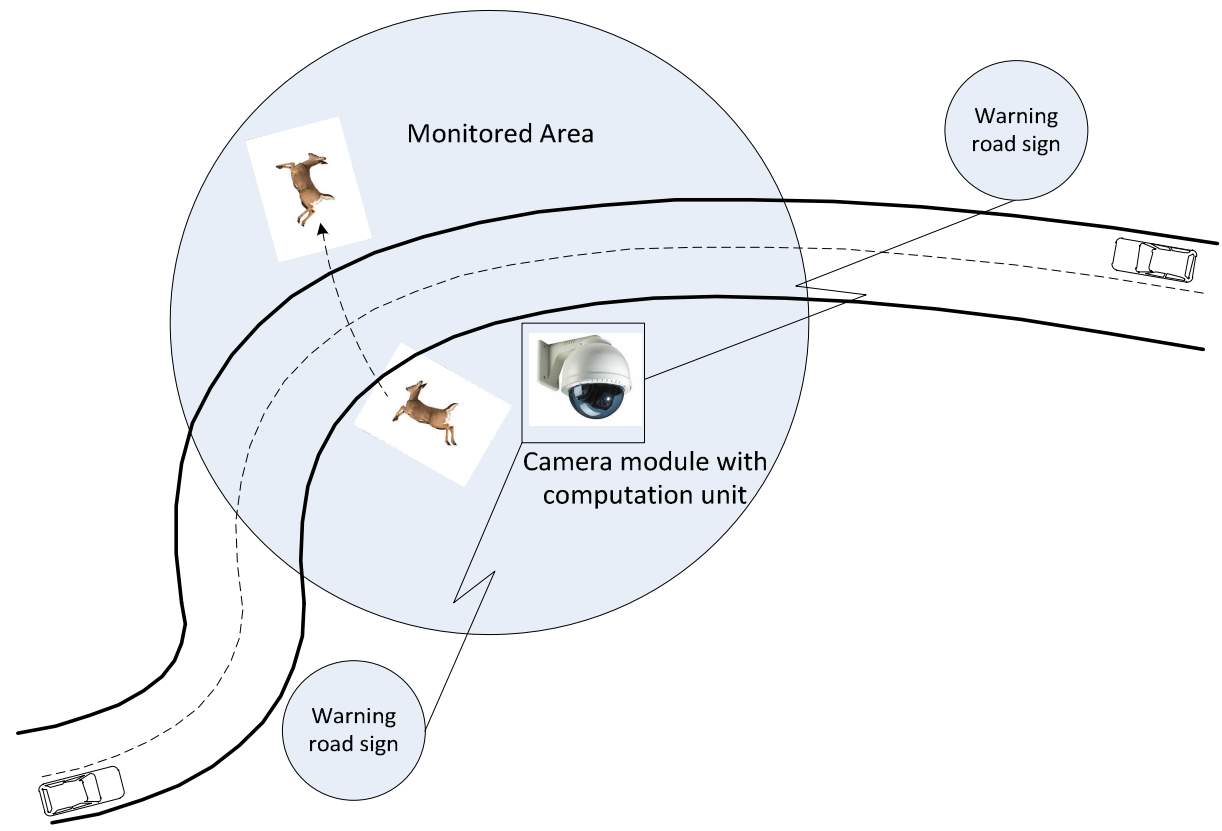

Fig. 1: The proposed system solution.

\section{Proposed software solution}

Proposed system consists of two main parts, training and testing part. During the training part, train database is used to create classification model, which is later used in the object recognition. For object representation were chosen local feature descriptors, like SIFT or SURF [6, 7]. Classification model is created based on the machine learning algorithm. In this system, combination of the bag of visual keypoints method and SVM witch radial base function were used. In the testing part, the video stream is captured and the algorithms of object detection and tracking are applied. The combination of background subtraction method and CAMShift algorithm were used. In the computer vision, one of the simplest ways how to separate moving object from scene background is to use background subtraction method. This method is widely used in computer vision and involves calculating a reference image, subtracting each new frame from the reference image; thresh holding and evaluating the result [8]. After successful determination and detection of region of the interest (ROI), CAMShift algorithm is applied to find optimal object size, position and orientation. Then the detected ROI is evaluated using object classification method and if this unknown ROI is classified as animal, which could be dangerous for the safety of the vehicle, the warning is displayed in the intelligent road signs.

\section{Experimental results}

In the experiments, we were focusing on the testing of the object detection and tracking part from the video sequences. Our proposed method for object detection and tracking follows the flow diagram shown in Fig. 2. 


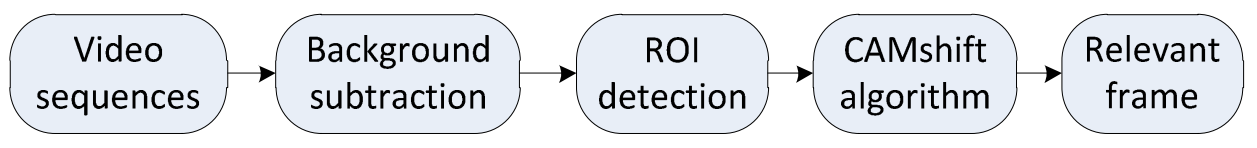

Fig. 2: Flow diagram of detecting and tracking method.

The experiments were done in $\mathrm{C}_{++}$language using OpenCV libraries. For the verification of the detection and tracking method robustness and accuracy, the method was tested on static video sequences with resolution $1920 \times 1080$. In video sequences, moving objects were represented by animals in their natural conditions or in zoo. In the video sequences, there were 5 kinds of big mammals, which can be dangerous for safety of the vehicle. These animal kinds are wolf, deer, brown bear, wild boar and fox. The examples of the animals from the database are shown in Fig. 3.
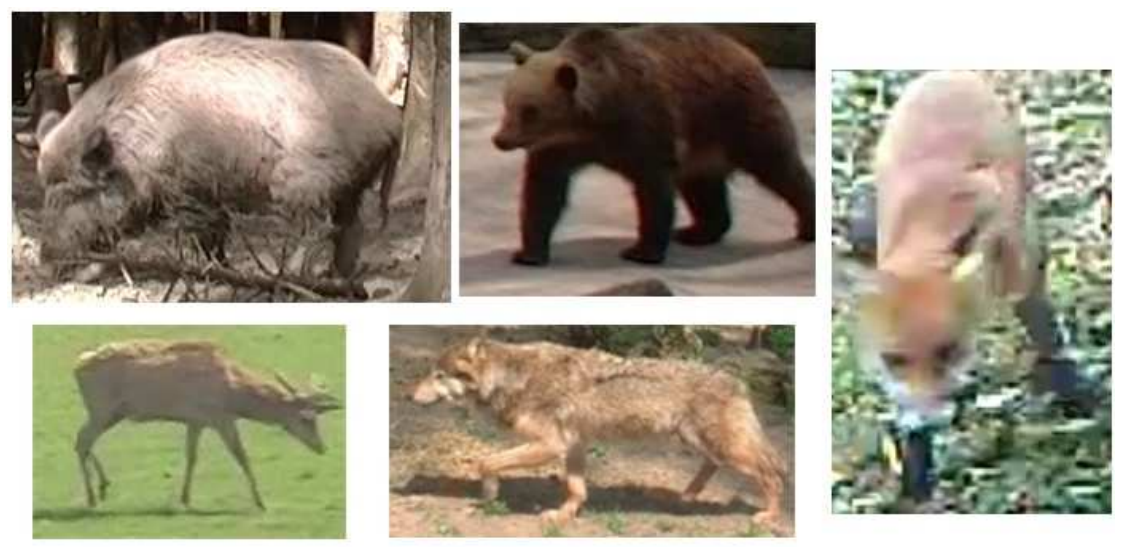

Fig. 3: Images from the database.

\subsection{Precision, Recall and F1 Measure}

Precision. Precision is defined as a proportion between the number of valid frames specified by the module and the number of all frames classified as valid frames. Precision is defined by formula 1:

$$
\text { Precision }=\frac{t_{p}}{t_{p}+f_{p}}
$$

where $t_{p}$ defines the number of frame correctly classified as valid class and $f_{p}$ defines the number of frame incorrectly classified as valid class.

Recall. Recall is defined as a proportion between the number of valid frames specified by the module and the number of all valid frames. Precision is defined by formula 2 :

$$
\text { Recall }=\frac{t_{p}}{t_{p}+f_{n}}
$$

where $t_{p}$ defines the number of frame correctly classified as valid class and $f_{n}$ defines the number of frames which were not classified as valid, but they should be.

F1 measure. F1 measure combines precision and recall in harmonic mean and F1 measure is defined by formula 3 :

$$
F 1=2 * \frac{\text { precision } * \text { recall }}{\text { precision }+ \text { recall }}
$$


Detector relevance. The detection and tracking method was also rated from the relevance point of view of the detected region of interest. The one image for every ROI was saved and according to these images, the detector relevance was calculated using formula 4 :

$$
\text { Relevance }=100 * \frac{\text { number of valid } R O I}{\text { whole number of } R O I} .
$$

The number of valid ROI was manually calculated using saved images from the ROIs.

\subsection{Results}

The method was tested on static video sequences with moving animal objects. The length of the tested videos per animal class is shown in Table 1. The recall-precision graph for tested video sequences is shown in Fig. 4.

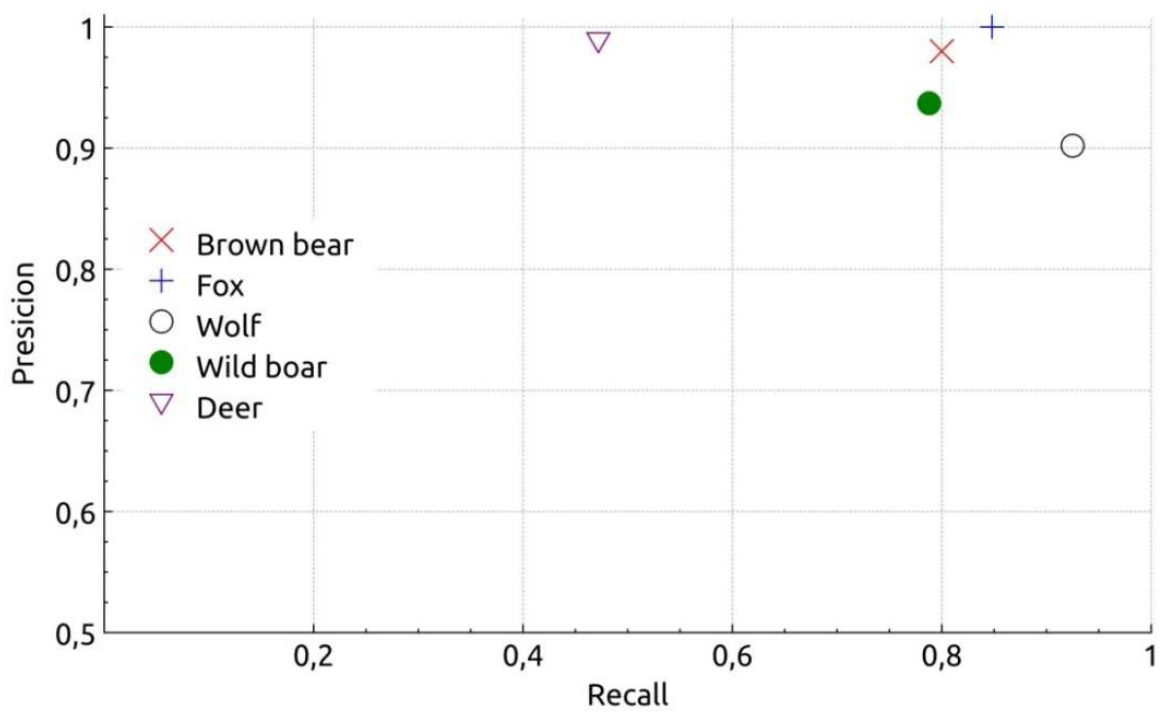

Fig. 4: Recall and precision.

According to the recall-precision graph, the best results for the particular classes are when their results are in the top right corner of the graph. From the given figure, it is evident that the results for classes fox, brown bear, wolf and wild boar were in the top-right corner of the graph. The low level of the recall was achieved for deer class. This was caused of the natural behaviour of the deer. The deer use to be static for a long period of time and in this case, detector will lose the animal objects. The relevance of the method, number of valid ROI and whole number of the ROI per animal class are shown in Table 1. The whole method relevance was at $87.05 \%$. In the case of the video sequences with brown bear and deer were achieved high values of detector relevance at $100 \%$, respectively $98.83 \%$. Detector relevance at $81.13 \%$ was achieved for class wild boar. In these videos, there were significantly changes in the illumination of the scene and in some cases, this caused a false detection. The similar score was achieved for video sequences with wolf. The lowest detector relevance was at $60.87 \%$ for fox class. The main reason of this is the false detection of the moving trees and its leaves, because of the strong wind.

Table 1: Video sequences information and detector relevance.

\begin{tabular}{|l|c|c|c|c|}
\hline & Video length [min] & Number of valid ROI & Whole number of ROI & $\begin{array}{c}\text { Detector } \\
\text { relevance }\end{array}$ \\
\hline Wild boar & 8,92 & 86 & 106 & 81,13 \\
\hline Fox & 1,35 & 14 & 23 & 60,87 \\
\hline Brown bear & 7,38 & 39 & 39 & 100,00 \\
\hline Wolf & 4,87 & 45 & 55 & 81,81 \\
\hline Deer & 9,40 & 85 & 86 & 98,83 \\
\hline Overall & $\mathbf{3 1 , 9 2}$ & $\mathbf{2 6 9}$ & $\mathbf{3 0 9}$ & $\mathbf{8 7 , 0 5}$ \\
\hline
\end{tabular}




\section{Conclusion and future work}

In this paper, the video camera road sign system of the early warning from collision with the wild animals was presented. The system consists of camera modules placed down the particularly chosen route and the intelligent road signs. The main idea of the proposed system was to help every driver on the road in the particular chosen areas. When the camera system detects the animal near or on the road and classifies this animal as dangerous for the safety of the vehicle, the warning is displayed on the intelligent road signs. In the experimental results were proven, that the system is capable of animal detection. The results can be even better, when we eliminate the false detection caused by the suddenly change of the scene illumination or moving trees and leaves in the scene. After successful object detection and tracking, the next step in the system is object classification. In this step, the animals in the scene need to be distinguished from the other moving objects, like cars, trucks or motorcycles. Then, the algorithms of machine learning (like Support Vector Machine) will be deployed to evaluate the animal class.

\section{Acknowledgement}

The work presented in the paper has been supported by the EUREKA project no. E! 6752 DETECTGAME: R\&D for Integrated Artificial Intelligent System for Detecting the Wildlife Migration.

\section{References}

[1] COLLINSON, P. A.: The application of camera based traffic monitoring systems. In: CCTV and Road Surveillance (Ref. No. 1999/126). IEE Seminar, p. 8/1 - 8/6, London. DOI: 10.1049/ic: 19990689.

[2] MAMMERI, A. - BOUKERCHE, A. - RENFEI, W. - JINGWEN, F.: Traffic sign detection, recognition and transmission system for smart vehicles. In: Global Communications Conference (GLOBECOM) IEEE 2013, December 2013, p. 4798 - 4803, Atlanta GA. DOI: 10.1109/GLOCOMW.2013.6855710.

[3] MAMMERI, A. - DEPU, Z. - BOUKERCHE, A. - ALMULLA, M.: An efficient animal detection system for smart cars using cascaded classifiers. In: Communications (ICC). IEEE 2014 International Conference, June 2014, p. 1854 - 1859, Sydney, NSW. DOI: 10.1109/ICC.2014.6883593.

[4] FORSLUND, D. - BJARKEFUR, J.: Night vision animal detection. In: Intelligent Vehicles Symposium Proceedings IEEE 2014, June 2014, p. 737 - 742, Dearborn, MI. DOI: 10.1109/IVS.2014.6856446.

[5] MUKHERJEE, A. - STOLPNER, S. - LIU, X. - VRENOZAJ, U. - FEI, C. - SINHA, A.: Large animal detection and continuous traffic monitoring on highways. In: Sensors. IEEE 2013, 3 - 6 November 2013, p. 1 - 3, Baltimore. ISSN 1930-0395, DOI: 10.1109/ICSENS.2013.6688330.

[6] LOWE, D.G.: Distinctive image features from scale-invariant keypoints. In: International Journal of Computer Vision. 2004, Vol. 60, No. 2, p. 91 - 110. ISSN: 0920-5691, DOI: 10.1023/B:VISI.0000029664.99615.94.

[7] BAY, H. - ESS, A. - TUYTELAARS, T.: SURF: Speeded Up Robust Features. In: Computer Vision and Image Understanding (CVIU). 2008, Vol. 110, No. 3, p. 346 - 359. ISSN: 1077-3142, DOI:10.1016/j.cviu.2007.09.014.

[8] KAEWTRAKULPONG, P. - BOWDEN, R.: An Improved Adaptive Background Mixture Model for Real-Time Tracking with Shadow Detection. In: Vision and Virtual Reality Group, 2nd European Workshop on Advanced Video Based Surveillance Systems AVBS01, September 2001.

[9] CONG, D. - SHI, P. - ZHOU, D.: An Improved Camshift Algorithm Based on RGB Histogram Equalization. In: Image and Signal Processing (CISP). 7th International Congress, $14-16$ October 2014, p. 426 - 430. DOI: 10.1109/CISP.2014.7003818. 